\title{
Importance of circulating tumor cells in newly diagnosed colorectal cancer
}

\author{
GUUS VAN DALUM ${ }^{1}$, GERRIT-JAN STAM ${ }^{1}$, LOES F.A. SCHOLTEN ${ }^{4}$, WALTER J.B. MASTBOOM ${ }^{2}$, \\ ISTVAN VERMES ${ }^{3}$, ARJAN G.J. TIBBE ${ }^{1}$, MARCO R. DE GROOT ${ }^{4}$ and LEON W.M.M. TERSTAPPEN ${ }^{1}$ \\ ${ }^{1}$ Department of Medical Cell BioPhysics, University of Twente, Enschede; Departments of ${ }^{2}$ Surgery, \\ ${ }^{3}$ Laboratory Medicine and ${ }^{4}$ Internal Medicine, Medisch Spectrum Twente, Enschede, The Netherlands
}

Received September 18, 2014; Accepted October 30, 2014

DOI: $10.3892 /$ ijo.2015.2824

\begin{abstract}
Presence of circulating tumor cells (CTC) is associated with poor prognosis in patients with metastatic colorectal cancer (CRC). The present study was conducted to determine if the presence of CTC prior to surgery and during follow-up in patients with newly diagnosed non-metastatic CRC can identify patients at risk for disease recurrence. In a prospective single center study 183 patients with newly diagnosed non-disseminated CRC, scheduled for surgery, were enrolled and followed-up for a median of 5.1 years. CTC were enumerated with the CellSearch system in 4 aliquots of $7.5 \mathrm{ml}$ of blood before surgery and at several time-points during followup after surgery. The results showed that $\geq 1 \mathrm{CTC} / 30 \mathrm{ml}$ of blood were detected in $44(24 \%)$ patients before surgery. Patients with CTC before surgery had a significant decrease in recurrence-free survival (RFS, log-rank test $\mathrm{P}=0.014$ ) and colon cancer related survival (CCRS, $\mathrm{P}=0.002$ ). The 5-year RFS dropped from 75 to $61 \%$ and the 5-year CCRS from 83 to $69 \%$ for patients with CTC before surgery. The presence of CTC and positive lymph nodes remained significant factors in multivariate analysis for recurrence-free survival (RFS). Surprisingly, the presence of CTC weeks after surgery was not significantly associated with RFS and CCRD whereas CTC 2-3 years after surgery was again significantly associated with RFS and CCRD. The presence of CTC in patients with stage I-III CRC before surgery is associated with a significant reduction in RFS and CCRS. These findings suggest a role of CTC detection to assess which patients need adjuvant treatment.
\end{abstract}

\section{Introduction}

The lifetime risk to develop colorectal carcinoma (CRC) is $>5 \%$ and because current therapy is not curative in all cases

Correspondence to: Professor Leon W.M.M. Terstappen, Department of Medical Cell BioPhysics, MIRA Institute, University of Twente, Drienerlolaan 5, P.O. Box 217, 7500 AE Enschede, The Netherlands

E-mail: 1.w.m.m.terstappen@utwente.nl

Key words: circulating tumor cells, colorectal cancer, colon cancer related death this disease is one of the leading causes of death worldwide (1). All curative resected CRC patients (30-50\%) will develop local recurrence or distant metastatic disease (2,3). Adjuvant chemotherapy is used in high risk patients, often defined as patients above stage IIB according to the TNM classification, angio-invasive growth, tumor perforation or obstruction and $<10$ detectable lymph nodes. Adjuvant chemotherapy results in a relative risk reduction of $\sim 30 \%$ in disease recurrence $(3,4)$. Because of a lack of $100 \%$ sensitivity and specificity of the known risk factors for disease recurrence, numerous patients receive adjuvant therapy without having presumed micrometastasis. On the other hand, a subgroup of patients classified as having a low risk for disease recurrence, thus, not receiving adjuvant treatment will develop disease recurrence. Better tools are necessary to discriminate between these patient groups.

The presence of tumor cells in blood of cancer patients may help to discriminate between these patient groups. The introduction of a validated system for the enumeration of circulating tumor cells (CTC) (5) enabled prospective clinical studies in both the metastatic and non-metastatic setting. Data from the multicenter prospective studies of CTC in metastatic breast (6), prostate (7) and colorectal (8) and a single center prospective study at diagnosis of breast cancer $(9,10)$ were reported earlier. These studies demonstrated that CTC are an independent predicting factor for disease-free survival and overall survival and these findings were confirmed by other studies (11-16). In the present study, we report on a single center prospective study in newly diagnosed CRC that was initiated at the same time as the original study in the metastatic colorectal cancer. The frequency of CTC in patients with metastatic colorectal cancer is extremely low, no CTC were detected with the CellSearch system in $52 \%$ of these patients using the FDA cleared protocol for $7.5 \mathrm{ml}$ of blood (8). In this study we investigate CTC in newly diagnosed patients without overt metastasis, the incidence of detectable CTC is expected to be lower and therefore, larger blood volumes will need to be analyzed to detect significant amount of CTC. To explore whether or not the presence of CTC in newly diagnosed patients could predict recurrence, $30 \mathrm{ml}$ of blood was analyzed for the presence of CTC before surgery, after surgery and at several time-points during a four-year follow-up. 


\section{Patients and methods}

Study design and patients. In this double blind single center cohort study 216 patients with colorectal malignancy and 58 patients with benign colorectal disease were enrolled at Medisch Spectrum Twente (MST), Enschede, the Netherlands. The ethics board of Medisch Spectrum Twente, approved the study protocol and all patients provided written informed consent. Patients were included between September 2003 and November 2008. Inclusion criteria were defined as patients aged $\geq 18$ years, newly diagnosed colorectal cancer without metastases and scheduled for surgery, ECOG performance state $0-1$. The main exclusion criterion was the presence of malignancy in the 5 years before inclusion in the medical history (excluding non-melanoma skin carcinoma or cervix carcinoma in situ). Thirty-three patients were excluded from analyses because of the following reasons: 15 patients were diagnosed with distant metastasis perioperative, 15 patients had a malignancy in their medical history, and 3 patients did not have CTC data at the inclusion time-point. This resulted in final cohort of 183 patients. The control group consisted of 58 patients undergoing colonoscopy or abdominal surgery in which no malignancy was detected. These patients were included throughout the study period to prevent bias in the staff performing the laboratory CTC analysis.

CTC enumeration during follow-up was done coinciding with a routine follow-up visit according to the Dutch guidelines for treatment of colorectal cancer (17). Treatment intention of all included patients with colorectal cancer was curative surgery. Peri-operative findings and pathologic outcome would define adjuvant therapy. Presence of CTC was blinded and did not influence adjuvant therapy. Patient records were reviewed in June of 2013 to record whether or not disease recurrence had occurred and if so when and whether or not the patient died and if this was related to colon cancer.

The primary end point of the study was to determine a correlation between the presence of CTC prior to surgery and recurrence-free survival (RFS). Secondary end points were defined as a correlation of CTC prior to surgery with colon cancer related death (CCRD) and correlation of blood draws during follow-up after surgery with RFS and CCRD.

Blood collection and CTC detection. Four peripheral blood samples were drawn by venipuncture into a $10-\mathrm{ml}$ CellSave Preservative tube (Veridex LLC, Raritan, NJ, USA). Timepoints of blood draw were before surgery or colonoscopy (Draw A), after surgery/before adjuvant therapy (Draw B), after adjuvant therapy (Draw C), after 1 year (Draw D), after 2 years (Draw E), after 3 years (Draw F) and after 4 years (Draw G). Four aliquots of $7.5 \mathrm{ml}$ were examined for the presence of CTC with the CellSearch system (Veridex). The CTC number was the total of the number found in the four aliquots. Analysis took place within $72 \mathrm{~h}$ after the blood draw. The CellSearch system enriched CTC using antibodies directed against the epithelial cell adhesion antigen (EpCAM) coupled to ferrofluids. The enriched cells were fluorescently labeled with the nucleic acid dye 4,6-diaminodino-2-phenylindole (DAPI) and phycoerythrin (PE) labeled monoclonal antibodies against cytokeratin 8,18 and 19 and allophycocyanin (APC) labeled antibodies directed against CD45. Images of CTC candidates were captured by the CellTracks Analyzer II and presented to experienced operators for classification and assigned as CTC when the objects were $>4 \mu \mathrm{m}$, stained with DAPI and cytokeratin, lacked CD45 and had morphological features consistent with that of a cell (5). The operators were blinded to the clinical status of the patient.

Statistical analysis. All patient data were collected in an Access database including demographic parameters such as age and gender and pathological findings including histological grade and TNM staging. Follow-up findings included recurrence date, adjuvant therapy and last outpatient control visit. The patient data was merged with the CTC enumeration at the moment of final analysis. Statistical analysis was performed using SPSS version 20.0 and $\mathrm{R}$ version 3.0.2 (18). A P-value $<0.05$ was considered to indicate a significant difference. All tests were two-sided. When dividing patients into a favorable and unfavorable group using CTC counts, unfavorable was considered one or more CTC. Kaplan Meier curves for RFS and CCRD were compared using the log-rank test. Betweengroup differences in categorical variables were tested by the Pearson's Chi-square test. The following significant univariate prognostic factors were included in a multivariate Cox proportional regression model: $\mathrm{T}$ stage, $\mathrm{N}$ stage and CTC status. Due to the low numbers of patients with $\mathrm{T} 1, \mathrm{~T} 1$ and $\mathrm{T} 2$ were grouped together in the multivariate model. The proportional hazard assumption was checked for all factors included in the model. Factors were removed from the multivariate model using stepwise elimination, using $\mathrm{P}>0.10$ as criteria.

\section{Results}

Patient characteristics and univariate analysis. The follow-up ranged from 1 to 109 months with a mean of 60 months and a median of 61 months. Recurrence of disease was observed in 48 of 183 (26\%) patients; 36 (20\%) patients died of causes related to colorectal cancer and $23(12 \%)$ patients died of other causes. The median follow-up of the patients alive at the end of the follow-up was 66 months. Patient characteristics and their relation with CTC, RFS and CCRD is shown in Table I. Univariate analysis showed a significant relationship between RFS and CCRD with T-stage, N stage and adjuvant therapy. The other variables: histological grade, tumor size and gender were not significant. T-stage $(\mathrm{P}=0.016)$ showed a significant difference in coincidence with unfavorable CTC counts.

Frequency of circulating tumor cells. In 44 out of the 183 patients (24\%) CTC were detected before surgery. This decreased to $29(19.9 \%)$ in the sample drawn weeks after surgery (and before the initiation of the adjuvant therapy when indicated). Ten of the 29 patients with CTC after surgery had CTC before surgery. The number of patients with one or more CTC found at the different time-points are provided in Table II.

Relation between circulating tumor cells and recurrence-free survival or colon cancer related death. Patients were divided into those with favorable (0 CTC) and unfavorable ( $\geq 1$ CTC) CTC for the different time-points. Table III shows the result of the log-rank test for each of the different blood draws for 
Table I. Characteristics of the 183 patients and their relation to recurrence-free survival (RFS) and colon cancer related death (CCRD) using a log-rank test.

\begin{tabular}{|c|c|c|c|c|c|}
\hline & $\mathrm{N}$ & $\%$ & $\begin{array}{c}\% \\
\text { CTC } \\
\geq 1\end{array}$ & $\begin{array}{c}\text { RFS } \\
\text { P-value }\end{array}$ & $\begin{array}{l}\text { CCRD } \\
\text { P-value }\end{array}$ \\
\hline T stage & & & & 0.008 & 0.001 \\
\hline $\mathrm{T} 4$ & 19 & 10 & $37^{\mathrm{a}}$ & & \\
\hline $\mathrm{T} 3$ & 108 & 59 & $28^{\mathrm{a}}$ & & \\
\hline $\mathrm{T} 2$ & 39 & 21 & $8^{\mathrm{a}}$ & & \\
\hline $\mathrm{T} 1$ & 13 & 7 & $8^{\mathrm{a}}$ & & \\
\hline Unkown & 4 & 2 & & & \\
\hline $\mathrm{N}$ stage & & & & 0.025 & 0.006 \\
\hline No & 112 & 61 & 19 & & \\
\hline N1 & 41 & 22 & 34 & & \\
\hline N2 & 26 & 14 & 27 & & \\
\hline Unkown & 4 & 2 & & & \\
\hline \multicolumn{6}{|l|}{ M stage } \\
\hline M0 & 183 & 100 & 24 & & \\
\hline M1 & 0 & & & & \\
\hline Histology grade & & & & 0.115 & 0.125 \\
\hline Poor & 11 & 7 & 36 & & \\
\hline Moderate & 133 & 73 & 25 & & \\
\hline Good & 13 & 6 & 8 & & \\
\hline Unkown & 26 & 14 & & & \\
\hline Adjuvant therapy & & & & $<0.001$ & $<0.001$ \\
\hline Yes & 54 & 30 & 23 & & \\
\hline No & 129 & 70 & 28 & & \\
\hline Gender & & & & 0.510 & 0.537 \\
\hline Male & 116 & 63 & 28 & & \\
\hline Female & 67 & 37 & 18 & & \\
\hline Continues & Mean & Min-max & & & \\
\hline Age & 66 & $37-85$ & & & \\
\hline Follow-up & 60 & $1-109$ & & & \\
\hline
\end{tabular}

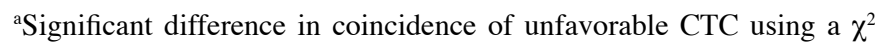
test.

RFS and CCRD. The number of patients participating in the follow-up CTC measurements decreased and to verify potential bias, the before surgery results of the same patients are provided in italics below each draw. When comparing unfavorable CTC to favorable CTC counts the risk of recurrence (Hazard ratio, 2.07, $\mathrm{P}=0.016$ ) and $\mathrm{CCRD}$ (Hazard ratio, $2.74, \mathrm{P}=0.003$ ) was significantly increased before surgery. The associated Kaplan-Meier curves for RFS and CCRD are shown in Fig. 1. The frequency of CTC 1-12 weeks after surgery decreased only slightly (from 24 to 20\%), but their presence was no longer significant, likewise CTC were not significant 1 year after surgery. Two and 3 years after surgery the presence of CTC again was highly significant for CCRD as shown in the Kaplan-Meier curves of Fig. 2. For the subgroup
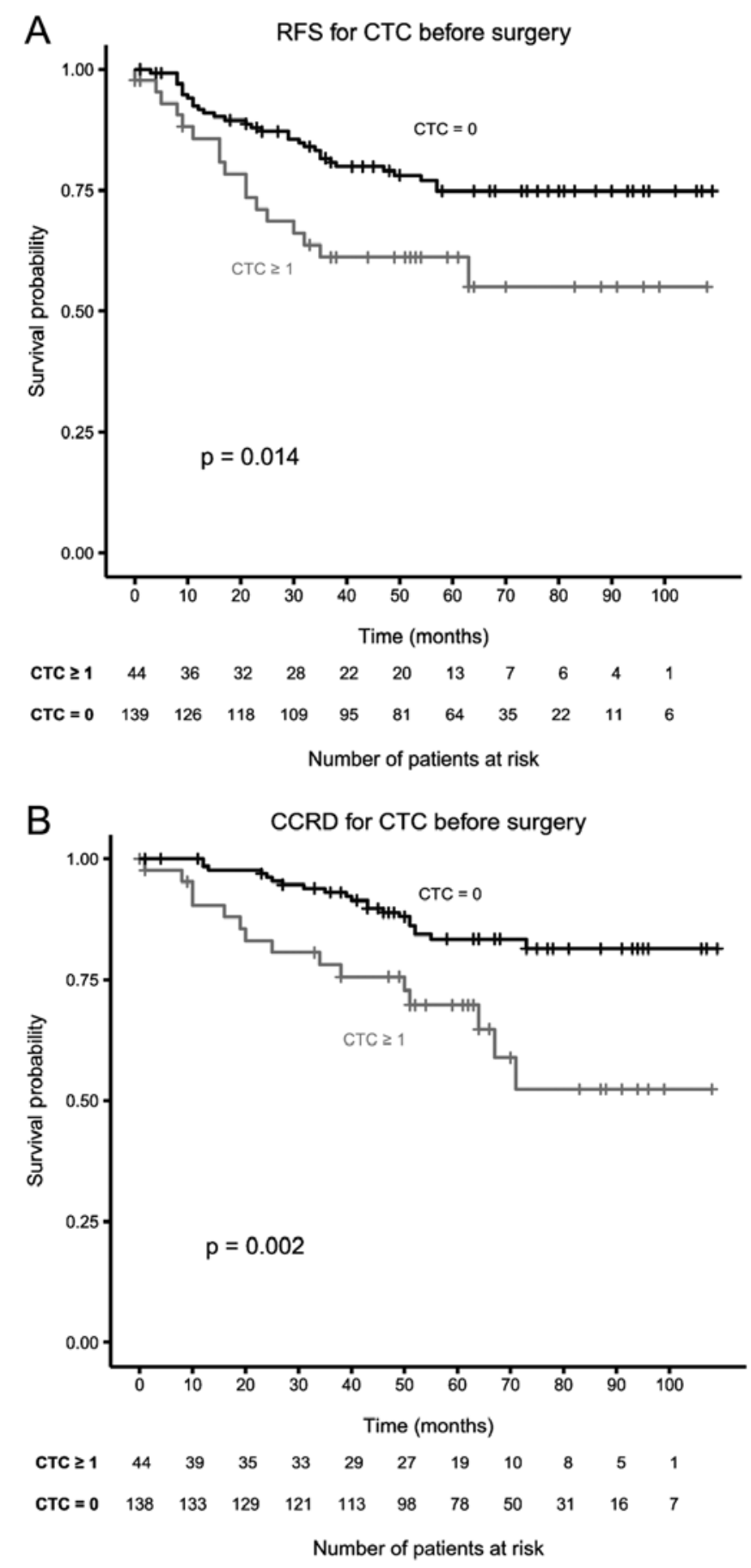

Figure 1. (A) Kaplan Meier graphs of recurrence-free survival and (B) colon cancer related death of colorectal cancer patients with $0 \mathrm{CTC} / 30 \mathrm{ml}$ and $>1$ $\mathrm{CTC} / 30 \mathrm{ml}$ of blood before surgical intervention.

of patients that received adjuvant therapy the presence of CTC before surgery and after completion of therapy was significant for RFS and CCRD, as listed in Table III. The subgroup of patients that did not receive adjuvant therapy (70\% of the 183 patients) did not have a significant relation between CTC and RFS $(\mathrm{P}=0.126)$ and CCRD $(\mathrm{P}=0.118)$ before surgery.

Multivariate analysis. Only the presence of CTC before surgery was included in the multivariate analysis. The univariate significant parameters CTC, N-stage and T-stage were 
Table II. Prevalence of circulating tumor cells before colon cancer surgery and at several time-points after surgery.

\begin{tabular}{|c|c|c|c|c|c|c|c|c|}
\hline CTCs & $\begin{array}{c}\text { Benign } \\
\text { disease } \\
\mathrm{N}=58 \\
\mathrm{n}(\%)\end{array}$ & $\begin{array}{c}\text { (A) Before } \\
\text { surgery } \\
n=183 \\
n(\%)\end{array}$ & $\begin{array}{c}\text { (B) After } \\
\text { surgery } \\
n=146 \\
n(\%)\end{array}$ & $\begin{array}{l}\text { (C) After adjuvant } \\
\text { therapy } \\
\mathrm{n}=42 \\
\mathrm{n}(\%)\end{array}$ & $\begin{array}{l}\text { (D) One year } \\
\text { after surgery } \\
n=116 \\
n(\%)\end{array}$ & $\begin{array}{l}\text { (E) Two years } \\
\text { after surgery } \\
n=82 \\
n(\%)\end{array}$ & $\begin{array}{l}\text { (F) Three years } \\
\text { after surgery } \\
n=47 \\
n(\%)\end{array}$ & $\begin{array}{l}\text { (G) Four years } \\
\text { after surgery } \\
n=16 \\
n(\%)\end{array}$ \\
\hline 0 & $50(86)$ & $139(76)$ & $117(80)$ & $32(76)$ & $96(83)$ & $74(90)$ & $42(89)$ & $13(81)$ \\
\hline$\geq 1$ & 8 (14) & $44(24)$ & $29(20)$ & $10(24)$ & $20(17)$ & $8(10)$ & $5(11)$ & 3 (19) \\
\hline 1 & 7 (12) & $28(15)$ & $17(12)$ & $6(14)$ & $11(9)$ & $3(4)$ & $3(6)$ & $2(13)$ \\
\hline 2 & $1(2)$ & $11(6)$ & $5(3)$ & $3(7)$ & $3(3)$ & $1(1)$ & $0(0)$ & $0(0)$ \\
\hline 3 & $0(0)$ & $0(0)$ & $2(1)$ & $0(0)$ & $0(0)$ & $1(1)$ & $0(0)$ & $0(0)$ \\
\hline 4 & $0(0)$ & $0(0)$ & $0(0)$ & $0(0)$ & $1(1)$ & $0(0)$ & $1(2)$ & $0(0)$ \\
\hline$>4$ & $0(0)$ & $5(3)$ & $5(3)$ & $1(2)$ & $5(4)$ & $3(4)$ & $1(2)$ & $1(6)$ \\
\hline
\end{tabular}

Table III. Relation between the presence of CTC and recurrence-free survival (RFS) or colon cancer related death (CCRD). For each time-point during follow-up (FU) the RFS and colon cancer related death (CCRD) for the before surgery draw of the same patients is shown in italics.

\begin{tabular}{lcccc}
\hline & $\mathrm{N}$ & Unfavorable $(\%)$ & RFS P-value & CCRD P-value \\
\hline Before surgery & 183 & 24 & 0.014 & 0.002 \\
After surgery & 143 & 20 & 0.940 & 0.425 \\
(before surgery draw) & & 22 & 0.252 & 0.029 \\
After adjuvant TX & 42 & 24 & 0.027 & 0.009 \\
(before surgery draw) & & 24 & 0.056 & 0.016 \\
After 1 year & 116 & 17 & 0.722 & 0.584 \\
(before surgery draw) & & 20 & 0.056 & 0.001 \\
After 2 years & 82 & 10 & 0.001 & $<0.001$ \\
(before surgery draw) & & 22 & 0.194 & 0.196 \\
After 3 years & 47 & 11 & 0.091 & 0.007 \\
(before surgery draw) & & 17 & 0.838 & 0.717 \\
After 4 years & 16 & 19 & 0.034 & 0.004 \\
(before surgery draw) & & 25 & 1.000 & 0.595 \\
\hline
\end{tabular}

also included in a Cox proportional hazards model. The multivariate regression was performed with a conditional stepwise elimination of the least significant parameters. For RFS and CCRD CTC and N-stage remained as significant predictors in the model (Table IV). In Fig. 3 both CTC and lymph nodes were combined to define at risk status in Kaplan-Meier curves for RFS and CCRD.

\section{Discussion}

Tumor cells present in the blood before surgical intervention reflect the invasion of the primary tumor in the bloodstream and these CTC have the potential to generate distant metastasis. Determination of CTC could be of value in determining which patients are at risk for recurrence after surgery and if validated could determine which patients need adjuvant treatment. The primary aim of the present study was to determine the number of CTC before surgery and correlate them to disease recurrence in patients with newly diagnosed CRC without distant metastatic disease at presentation or perioperatively. Secondary endpoints were to determine the correlation between CTC presence and recurrence-free survival, colon cancer related death before and during 4 years of follow-up after surgery.

Our results showed a significant correlation between the presence of CTC before surgery and RFS and CCRD. In multivariate comparison of established risk factors determined perioperatively, only the $\mathrm{N}$-stage and the presence of CTC before surgery remained significant for both RFS and CCRD (Table IV). Presence of both lymph node involvement and CTC clearly identifies a subgroup of patients with extremely high risk for disease recurrence and death from colon cancer indicating that current treatment is not sufficient for this group of patients. The presence of only one of those risk factors was much less predictive (Figs. 1 and 3). Separation of the patients into those who received and those who did not receive adjuvant therapy showed that the presence of CTC before surgery was only significant for those patients who did receive adjuvant therapy. The latter suggests a role for CTC, 
A
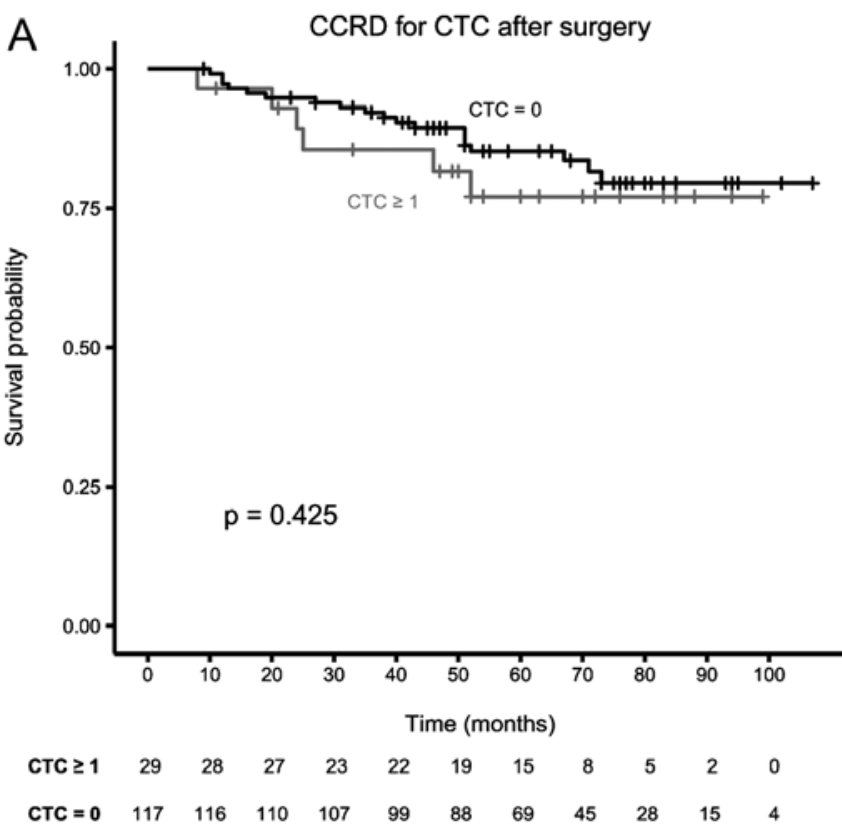

Number of patients at risk

C

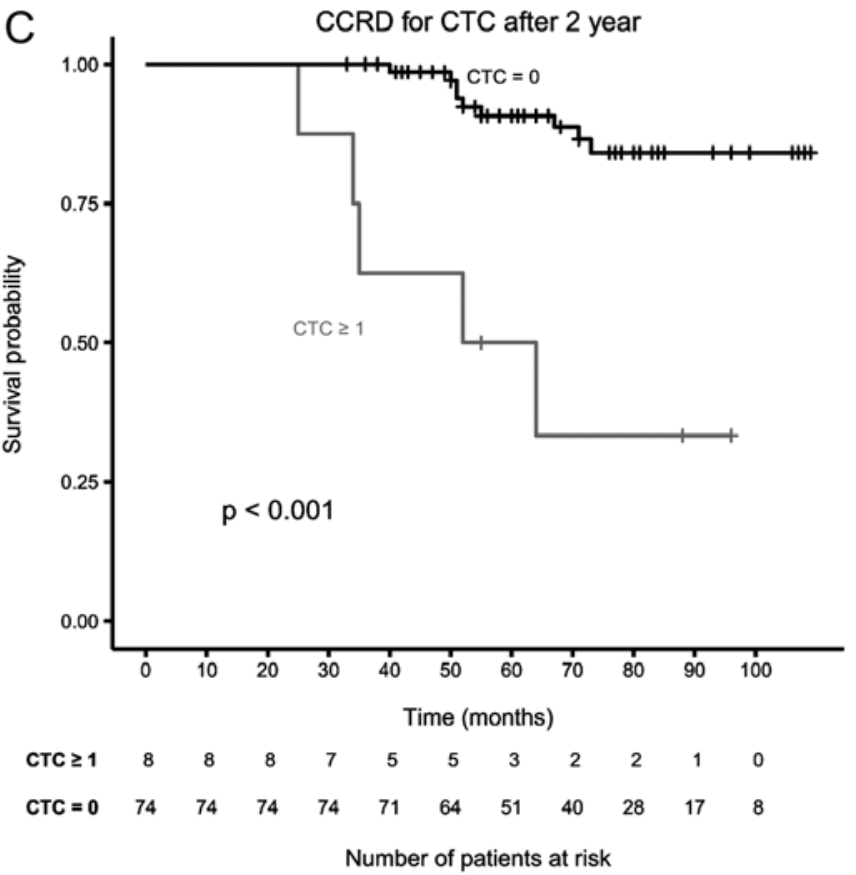

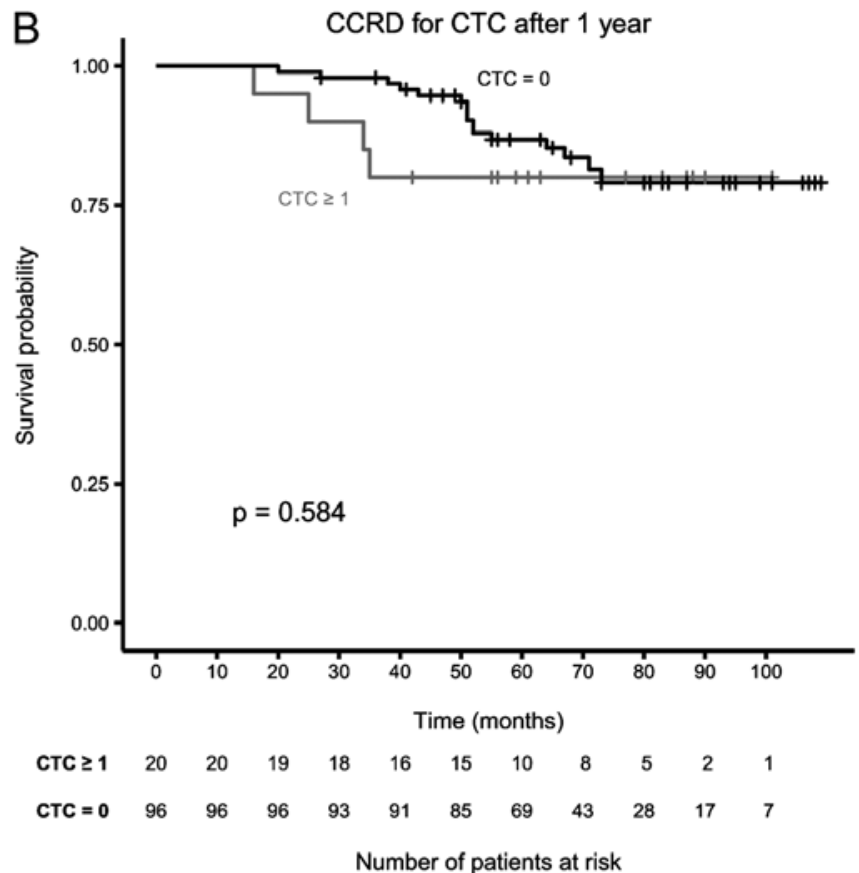

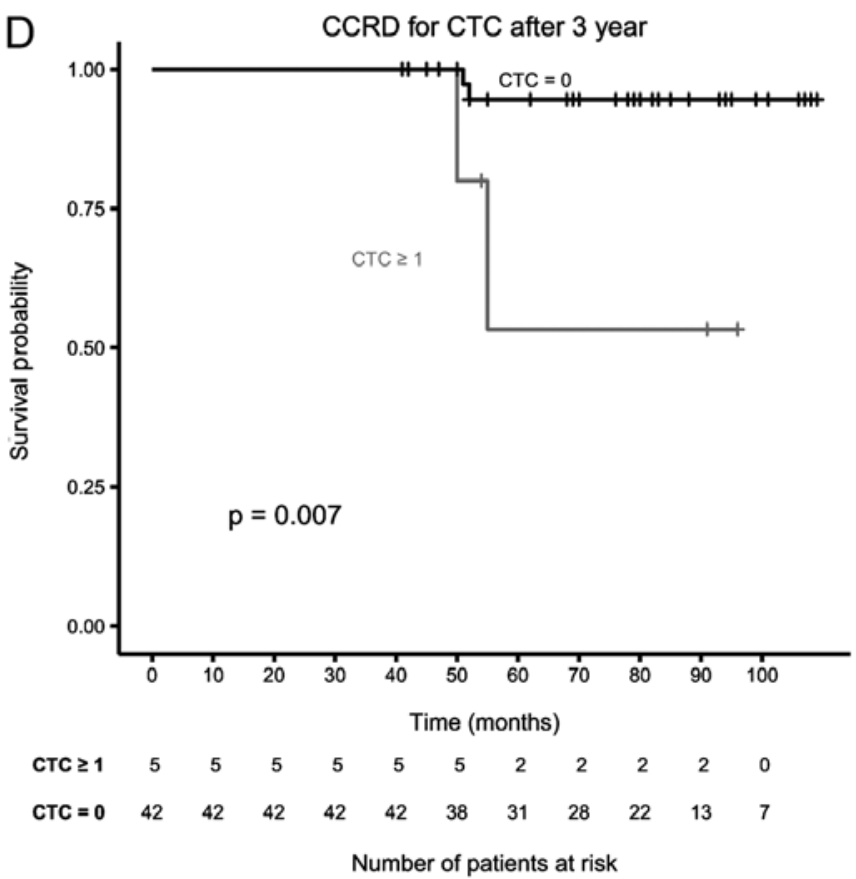

Figure 2. Kaplan Meier graphs of colon cancer related death of colorectal cancer patients with 0 CTC/30 ml and >1 CTC/30 ml of blood after (A) surgery, (B) 1 year after surgery, (C) 2 years after surgery and (D) 3 years after surgery.

Table IV. Multivariate Cox proportional hazard regression analysis of all univariate significant parameters with a conditional stepwise elimination of least significant parameters for recurrence-free survival (RFS) and colon cancer related death (CCRD).

\begin{tabular}{lccccr}
\hline & Variables & Categories & P-value & Hazard ratio & 95\% CI \\
\hline RFS & CTC & Yes vs. no & 0.030 & 1.96 & $1.06-3.61$ \\
& N-Stage & N0 vs. N1 & 0.021 & 2.21 & $1.12-4.08$ \\
& N-Stage & N0 vs. N2 & 0.084 & 2.12 & $0.91-4.59$ \\
CCRD & CTC & Yes vs. no & 0.002 & 2.88 & $1.46-5.66$ \\
& N-Stage & N0 vs. N1 & 0.007 & 2.78 & $1.32-5.84$ \\
& N-Stage & N0 vs. N2 & 0.034 & 2.68 & $1.08-6.65$ \\
\hline
\end{tabular}



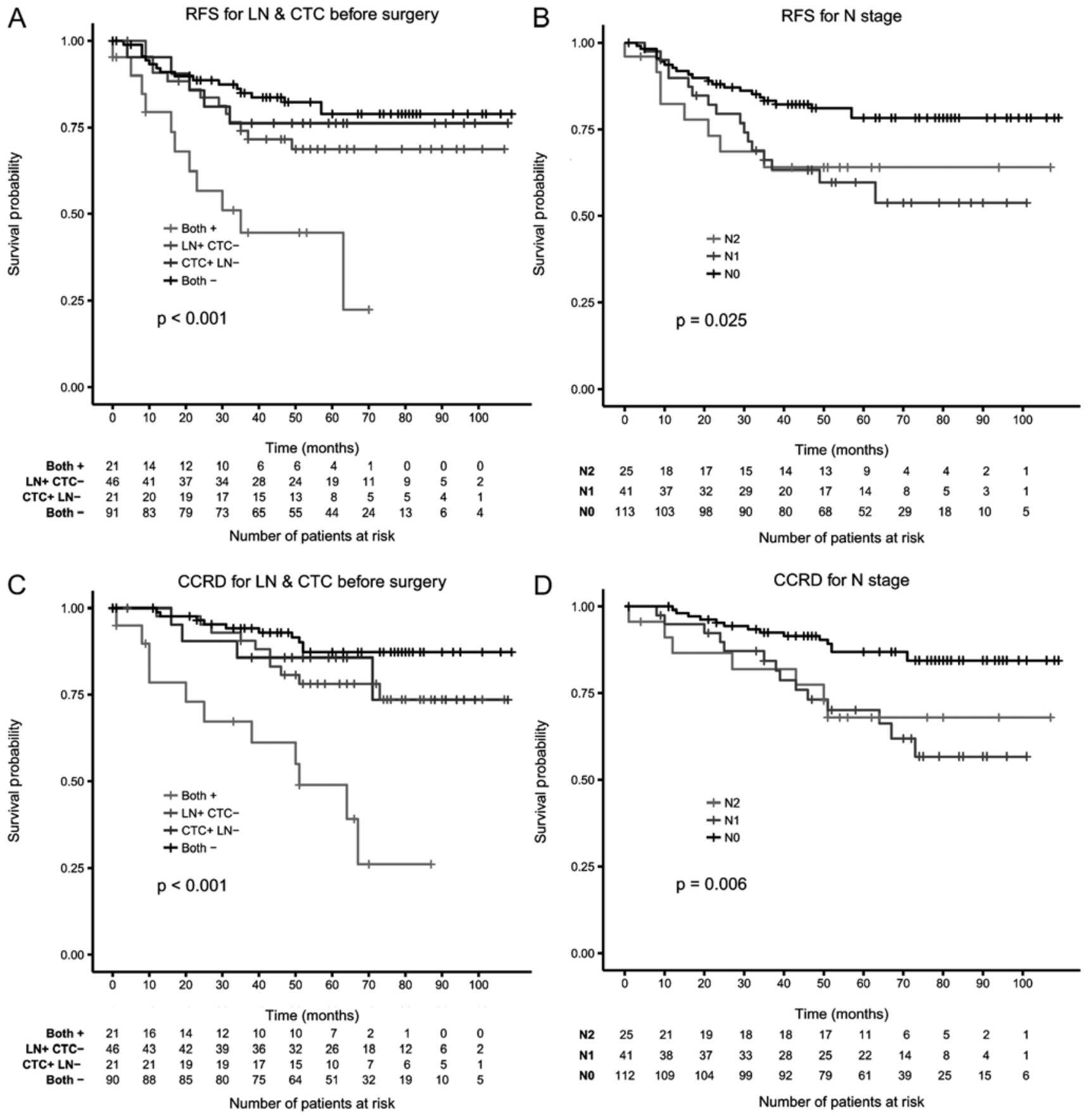

Figure 3. Kaplan-Meier graphs of recurrence-free survival (A and B) and colon cancer related death (C and D) of colorectal cancer patients with $0 \mathrm{CTC} / 30 \mathrm{ml}$ and $>1 \mathrm{CTC} / 30 \mathrm{ml}$ of blood together with positive and negative lymphnodes. (B and D) The results of only lymph node status.

suggesting a more aggressive therapy rather than providing therapy to those patients that currently are not receiving adjuvant therapy. The presence of CTC during follow-up in the group of patients without CTC before surgery and not receiving adjuvant therapy suggests that the sensitivity of the CellTracks technology for the detection of CTC in this group of patients before surgery is insufficient.

The significant relation between the presence of CTC and the potential for disease recurrence has also been shown for patients undergoing surgery for primary breast cancer $(9,10,14,19,20)$. The reported frequency of CTC in these studies are quite similar and a model predicted that the sensitivity of CTC detection will need to reach $9 \pm 6 \mathrm{CTC} / 1$ blood to detect a tumor before the formation of distant metastasis (21). Our observations on the significant association between the presence of CTC and lower progression free survival and death is in agreement with those in metastatic colorectal cancer $(8,13,22)$. The prevalence of CTC detected by the CellSearch system in $7.5 \mathrm{ml}$ of blood in metastatic colorectal cancer is low. To increase the possibility to find CTC in early colorectal cancer the volume of blood was increased from 7.5 to $30 \mathrm{ml}$ by running four CellSearch tests in parallel. Based on statistical analysis it was already suggested that the presence of $\geq 1 \mathrm{CTC}$ was associated with a poor outcome (23). Based on the low prevalence and the possible significance of 1 CTC the cut-off point was set at $\geq 1 \mathrm{CTC}$ in $30 \mathrm{ml}$ of blood. The low prevalence 
of CTC detected by the CellSearch system in non-metastatic colorectal cancer has been confirmed in other studies (24-26). The variation in CTC frequency in these reports can likely be contributed to the inclusion of different stages of the disease and the absence in most studies of random inclusion of normal samples during the study period. For non-metastatic colon cancer low numbers of CTCs in $7.5 \mathrm{ml}$ have been correlated to N-stage in other studies (27). We found no correlation to $\mathrm{N}$-stage but did find a correlation to T-stage.

In a recent study, the CTC frequency from patients with metastatic colorectal, prostate and breast cancer was modeled and predicted that $99 \%$ of patients had at least one CTC in 5 liters of blood before initiation of therapy for metastatic disease (28). In the same report each 10 -fold increase in CTC was associated with a 6.6-month decrease in survival (28). To detect CTC in all patients with micro and or macro metastatic disease the volume of blood analyzed will need to be increased substantially and the specificity of detection improved. An increase in blood volume, however, will also result in a higher false positive number of CTC. In the present study 7 patients with benign colorectal disease had one and 1 patient had two CTC detected in $30 \mathrm{ml}$ of blood. Detection of cells with the CTC phenotype in $7.5 \mathrm{ml}$ of blood in patients with benign colorectal disease using the CellSearch system and other systems have also been reported by others (29). Whether or not these cells are indeed tumor cells or are an artifact of the current detection technologies will need to be investigated. Surprisingly, the presence of CTC from blood drawn 4-92 (28 mean) days and 1 year after surgery was not significantly related to RFS and CCRD. In contrast, the presence of CTC from blood drawn 2, 3 and 4 years after surgery was significantly correlated with RFS and CCRD (except after 3 years for RFS). CTC measured before surgery from the same patient group was not significantly related to RFS and CCRD. This suggests that residual disease directly after surgery +/- adjuvant therapy and one year later while in situ and progressing is shedding tumor cells into the peripheral blood and for those patients that ultimately recur the shedding of tumor cells by the micro-metastasis until year two after surgery is not sufficient to be detected with the CTC detection technology used in the present study. An alternative explanation for the presence of CTC after surgery that are not related to outcome rises from the observation that a large number of CTC are released during surgery (30). These tumor cells may just not have the potential to form distant metastasis. The number of approaches available for CTC detection has grown since the start of the present study (31), evaluation of these different approaches in controlled clinical studies will show whether the advances are sufficient to detect tumor cells in all patients with disseminated disease. The latter will allow treatment on the basis of presence of disease rather than on an increased risk profile.

In summary, the present study demonstrates that the presence of CTC in patients with non-metastatic CRC before surgery is associated with a statistically significant higher risk of disease recurrence and shorter recurrence-free survival and a higher colon cancer related death. Presence of CTC also has a significant impact on the disease course when measured 2 to 4 years after surgery but not within the first year after surgery. Further improvement of sensitivity and specificity of CTC detection technology is warranted as it has the promise to be incorporated in the diagnostic armamentarium to determine which patients do and do not need adjuvant therapy.

\section{Acknowledgements}

The present study was supported by the Immunicon Corporation, responsible for the development of the CellSearch system.

\section{References}

1. Jemal A, Siegel R, Ward E, Hao Y, Xu J, Murray T and Thun MJ: Cancer statistics, 2008. CA Cancer J Clin 58: 71-96, 2008.

2. Shah S, Haddad R and Al-Sukhni W: Surgical resection of hepatic and pulmonary metastases from colorectal carcinoma. J Am Coll Surg 202: 468-475, 2006.

3. Safi F and Beyer HG: The value of follow-up after curative surgery of colorectal carcinoma. Cancer Detect Prev 17: 417-424, 1993.

4. André T, Boni C, Navarro M, et al: Improved overall survival with oxaliplatin, fluorouracil, and leucovorin as adjuvant treatment in stage II or III colon cancer in the MOSAIC trial. J Clin Oncol 27: 3109-3016, 2009.

5. Allard WJ, Matera J, Miller MC, et al: Tumor cells circulate in the peripheral blood of all major carcinomas but not in healthy subjects or patients with nonmalignant diseases. Clin Cancer Res 10: 6897-6904, 2004.

6. Cristofanilli M and Budd G: Circulating tumor cells, disease progression, and survival in metastatic breast cancer. Engl J Med 351: 781-791, 2004.

7. De Bono JS, Scher HI, Montgomery RB, et al: Circulating tumor cells predict survival benefit from treatment in metastatic castrationresistant prostate cancer. Clin Cancer Res 14: 6302-6309, 2008.

8. Cohen SJ, Punt CJA, Iannotti N, et al: Relationship of circulating tumor cells to tumor response, progression-free survival, and overall survival in patients with metastatic colorectal cancer. J Clin Oncol 26: 3213-3221, 2008.

9. Franken B, de Groot MR, Mastboom WJB, Vermes I, van der Palen J, Tibbe AGJ and Terstappen LWMM: Circulating tumor cells, disease recurrence and survival in newly diagnosed breast cancer. Breast Cancer Res 14: R133, 2012.

10. Dalum G van, Stam GJ van der, Tibbe AGJ, et al: Circulating tumor cells before and during follow-up after breast cancer surgery. Int J Oncol 46: 407-413, 2015.

11. Liu MC, Shields PG, Warren RD, et al: Circulating tumor cells: a useful predictor of treatment efficacy in metastatic breast cancer. J Clin Oncol 27: 5153-5159, 2009.

12. Pierga JY, Hajage D, Bachelot T, et al: High independent prognostic and predictive value of circulating tumor cells compared with serum tumor markers in a large prospective trial in first-line chemotherapy for metastatic breast cancer patients. Ann Oncol 23: 618-624, 2012.

13. Tol J, Koopman M, Miller MC, et al: Circulating tumour cells early predict progression-free and overall survival in advanced colorectal cancer patients treated with chemotherapy and targeted agents. Ann Oncol 21: 1006-1012, 2010.

14. Lucci A, Hall CS, Lodhi AK, et al: Circulating tumour cells in non-metastatic breast cancer: a prospective study. Lancet Oncol 13: 688-695, 2012.

15. Bidard FC, Mathiot C, Delaloge S, et al: Single circulating tumor cell detection and overall survival in non-metastatic breast cancer. Ann Oncol 21: 729-733, 2010.

16. Martín M, Custodio S, de Las Casas MLM, et al: Circulating tumor cells following first chemotherapy cycle: an early and strong predictor of outcome in patients with metastatic breast cancer. Oncologist 18: 917-923, 2013.

17. www.oncoline.nl. Integr Kankercent Ned, 2013.

18. R Core Team: R: A Language and Environment for Statistical Computing, 2013.

19. Jueckstock JK, Rack BK, Zwingers T,Hepp PGM, Schneeweiss A, Beckmann MW, Lichtenegger W, Sommer HL, Pantel K, Tesch H, Forstbauer H, Lorenz R, Rezai M, Neugebauer JK, Andergassen U, Friese K and Janni W: Prognostic relevance of circulating tumor cells (CTC) before adjuvant chemotherapy in patients with breast cancer: results of the German SUCCESS trial. J Clin Oncol 29 (Suppl): 1033, 2011. 
20. Pierga JY, Bidard FC, Mathiot C, et al: Circulating tumor cell detection predicts early metastatic relapse after neoadjuvant chemotherapy in large operable and locally advanced breast cancer in a phase II randomized trial. Clin Cancer Res 14: 7004-7010, 2008.

21. Coumans FA, Siesling S and Terstappen LW: Detection of cancer before distant metastasis. BMC Cancer 13: 283, 2013.

22. Cohen SJ, Punt CJ, Iannotti N, et al: Prognostic significance of circulating tumor cells in patients with metastatic colorectal cancer. Ann Oncol 20: 1223-1229, 2009.

23. Tibbe AGJ, Miller MC and Terstappen LWMM: Statistical considerations for enumeration of circulating tumor cells. Cytometry A 71: 154-162, 2007.

24. Thorsteinsson M, Soletormos G and Jess P: Low number of detectable circulating tumor cells in non-metastatic colon cancer. Anticancer Res 31: 613-617, 2011.

25. Sastre J, Maestro ML, Puente J, et al: Circulating tumor cells in colorectal cancer: correlation with clinical and pathological variables. Ann Oncol 19: 935-938, 2008.
26. Hiraiwa $\mathrm{K}$, Takeuchi $\mathrm{H}$, Hasegawa $\mathrm{H}$, et al: Clinical significance of circulating tumor cells in blood from patients with gastrointestinal cancers. Ann Surg Oncol 15: 3092-3100, 2008

27. Gazzaniga P, Gianni W and Raimondi C: Circulating tumor cells in high-risk nonmetastatic colorectal cancer. Tumor Biol 34: 2507-2509, 2013

28. Coumans FA, Ligthart ST, Uhr JW and Terstappen LWMM: Challenges in the enumeration and phenotyping of CTC. Clin Cancer Res 18: 5711-5718, 2012.

29. Pantel K, Denève E, Nocca D, et al: Circulating epithelial cells in patients with benign colon diseases. Clin Chem 58: 936-940, 2012.

30. Wind J, Tuynman JB, Tibbe AG, Swennenhuis JF, Richel DJ, van Berge Henegouwen MI and Bemelman WA: Circulating tumour cells during laparoscopic and open surgery for primary colonic cancer in portal and peripheral blood. Eur J Surg Oncol 35: 942-950, 2009

31. Bednarz-Knoll N, Alix-Panabières C and Pantel K: Clinical relevance and biology of circulating tumor cells. Breast Cancer Res 13: 228, 2011. 